\title{
Editorials
}

\section{Pain management after ambulatory surgery - Where is the disconnect?}

\author{
Paul F. White PhD MD FANZCA
}

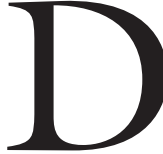
ESPITE recent advances in our knowledge of multimodal analgesic therapies for pain management, ${ }^{1}$ many patients undergoing ambulatory surgery continue to experience unacceptable levels of pain after their operations. $^{2-4}$ Postoperative pain is a complex and multi-factorial symptom which requires a thoughtful approach using a variety of treatment modalities to obtain optimal outcomes with respect to patient comfort and facilitating the recovery process. Multimodal (or "balanced") analgesia represents an approach to preventing postoperative pain that involves administering a combination of opioid and non-opioid analgesics, that act at different sites within the central and peripheral nervous systems, in an effort to eliminate opioidrelated side effects. ${ }^{1,5}$ The current armamentarium of analgesic drugs and techniques for the management of postoperative pain continues to grow at a rapid rate. However, there appears to be a significant 'disconnect' between the publication of analgesic studies in the peer-reviewed literature demonstrating approaches to improving acute pain management and the application of these concepts in clinical practice. ${ }^{6}$

A part of the problem relates to the increasing number and complexity of elective operations that are being performed on an ambulatory (or short-stay) basis, where the use of conventional opioid-based intravenous patient-controlled analgesia (PCA) and central neuroaxial (spinal and epidural) analgesia techniques are simply not practical for acute pain management. This rapidly expanding patient population requires an aggressive perioperative analgesic regimen that provides effective pain relief, has minimal side effects, is intrinsically safe, and can be managed by patients and their family members away from a hospital or surgical centre.

The adequacy of postoperative pain control is one of the most important factors in determining when a patient can be safely discharged from a surgical facility, and also has a major influence on the patient's ability to resume his/her normal activities of daily living. ${ }^{3}$ Perioperative analgesia has traditionally been provided using potent opioid (narcotic) analgesics. However, extensive reliance on opioid medication for acute pain management is associated with a variety of perioperative complications [e.g., ventilatory depression, drowsiness and sedation, postoperative nausea and vomiting (PONV), pruritus, urinary retention, ileus, constipation] which can contribute to a delayed hospital discharge and resumption of normal activities of daily living. ${ }^{6}$ Anesthesiologists are increasingly turning to the use of a combination of non-opioid analgesic medications as the 'first line' of therapy for the prevention of pain in the postoperative period. However, opioid analgesics will likely remain the primary treatment option for patients who require "rescue" analgesic therapy in the postoperative period, until more potent and rapid-acting non-opioid analgesics become available for routine clinical use.

In 2000, the Joint Commission on Accreditation of Healthcare Organizations (JCAHO) introduced new standards that mandated pain assessment and treatment as part of routine patient care, in an attempt to improve control of acute pain. Unfortunately, many medical institutions have misinterpreted this mandate as requiring that the treatment of pain must be

CAN J ANESTH 2008/55: 4 / pp 201-207

From Department of Anesthesiology and Pain Management, University of Texas Southwestern Medical Center at Dallas, Dallas, Texas, USA.

Address correspondence to: Dr. Paul F. White, Department of Anesthesiology and Pain Management, UT Southwestern Medical Center, 5323 Harry Hines Boulevard, Dallas, TX 75390-9068; USA. Phone: 214-648-6403; Fax: 214-648-2920; E-mail: paul. white@utsouthwestern.edu

Financial support: This work was supported in part by the Margaret Milam McDermott Distinguished Chair in Anesthesiology and the White Mountain Institute in Los Altos, CA (Dr. White is the President). 
guided by patient reports of pain intensity, indexed to a numerical pain scale. ${ }^{7}$ Following the implementation of a routine numeric pain scoring system in their recovery room, Frasco et al. ${ }^{8}$ reported a significant increase in the use of opioid analgesics. Vila et al. ${ }^{9}$ reported that as a result of the JCAHO-mandated policy for pain management, the incidence of opioidrelated adverse reactions increased from 11 to 25 per 100,000 inpatient days at their medical centre. The majority of adverse drug reactions were preceded by a documented decrease in the patient's level of consciousness due to opioid-related sedation. In the ambulatory setting, the primary factor responsible for post-discharge nausea and vomiting is the oral use of oral opioid-containing analgesics. ${ }^{10}$ Compared to the use of an oral combination of acetaminophen and codeine, Raeder et al. ${ }^{11}$ reported that use of ibuprofen was associated with fewer gastrointestinal side effects (e.g., PONV, constipation) after ambulatory surgery.

Observational studies have confirmed that poorly controlled pain is a major factor contributing to a delayed discharge after ambulatory surgery. ${ }^{2,4}$ Improving postoperative pain control will accelerate normalization of the patient's quality of life and resumption of normal functionality. ${ }^{5}$ It has been suggested that the key to improving the recovery process after ambulatory surgery is the adaptation of multimodal (or "balanced") analgesia techniques as the standard approach for the prevention and treatment of pain in the ambulatory setting. ${ }^{1}$ Early studies evaluating approaches to facilitating the recovery process have demonstrated that the use of multimodal analgesic techniques can improve early recovery, as well as other clinically-meaningful outcomes after ambulatory surgery. ${ }^{12,13}$ These benefits have been confirmed in more recent studies, ${ }^{14,15}$ and multimodal techniques are the currently recommended practice in fast-track clinical care plans. ${ }^{5}$ It is clear that the reliance on a single non-opioid analgesic modality [e.g., local analgesics, non-steroidal anti-inflammatory drugs (NSAIDs) and/or acetaminophen] will not suffice to control moderate-severe postoperative pain, and excessive reliance on opioid analgesics produces too many undesirable side effects. ${ }^{6,7}$

The short and long term benefits of using multimodal analgesia regimens to reduce opioid-related side effects remain somewhat controversial because the definition of "multimodal analgesia" is not uniform in the anesthesia and surgery literature. ${ }^{1}$ In some contexts, multimodal analgesia refers to systemic administration of analgesic drugs with different mechanisms of action, while in other situations, it refers to concurrent application of analgesic pharmacotherapy in combination with regional analgesia. However, the major deficiency with many of the published studies is that the multimodal analgesic therapies were not continued into the post-discharge period. ${ }^{16}$ For example, perioperative administration of the COX-2 inhibitors as part of a multi-modal analgesic regimen in outpatients undergoing inguinal hernia repair provided limited benefit beyond the early postoperative period. ${ }^{17}$ However, when the COX-2 inhibitors are administered for three-to-five days after ambulatory surgery, ${ }^{14,15}$ the benefits with respect to clinicallyrelevant patient outcomes extended well beyond the simple (and predictable) reduction in pain and the need for opioid analgesic medications.

While opioid analgesics will continue to play an important role in the acute treatment of moderate-to-severe pain in the early postoperative period, non-opioid analgesics will likely assume a greater role as "preventative" analgesics in the future, as the number of minimally-invasive ("key hole") surgery cases continues to expand. In addition to local anesthetics and traditional NSAIDs, COX-2 inhibitors, acetaminophen, ketamine, dextromethorphan, alpha-2 agonists, gabapentin, pregabalin, beta-blockers, and glucocorticoid steroids can all provide beneficial effects when administered in appropriate doses, as part of a multimodal analgesic regimen in the perioperative period. ${ }^{1,6}$ Although the local anesthetic effects of lidocaine are well-known, the potential beneficial effects of intravenous lidocaine in reducing postoperative pain and facilitating the recovery process has only recently been described in the anesthesia literature. ${ }^{18}$ Novel compounds like capsicum have also been found to produce prolonged analgesic effects, resulting from their ability to alter nociceptive input at the peripheral nerve ending. ${ }^{19}$

Preemptive analgesic techniques have been postulated to provide superior analgesia by preventing the establishment of central sensitization. ${ }^{20}$ Unfortunately, this approach does not appear to offer any clinically significant advantages over so-called preventative multimodal analgesic regimens, when an effective approach to pain management is initiated in the early postoperative period and extended into the post-discharge period. ${ }^{21}$ The recent study by Sun $e t a l .{ }^{21}$ failed to find any additional benefit to giving a preoperative dose of the COX-2 inhibitor celecoxib, when compared to simply giving the drug for preventative analgesia in the postoperative period. Extending a multimodal analgesic treatment regimen into the postoperative (and post-discharge) period is clearly more important for improving pain management than just relying on preemptive analgesic techniques. 
The need to educate patients and their family members (caregivers) about the importance of continuing their analgesic medications after they leave the hospital and/or day-surgery centre, is of critical importance to improving the quality of pain control and facilitating recovery in the future. The recent attention given to opioid-related side effects as impediments to achieving a high degree of patient satisfaction and early discharge home after surgery has also increased interest in using local (and regional anesthetic) techniques during and after ambulatory surgery. ${ }^{6,7}$ For example, the use of continuous local anesthetic techniques (e.g., perineural blocks, wound infiltration) has become increasingly popular, due to their ability to control moderate-to-severe pain after major ambulatory surgery procedures. ${ }^{22-25}$ The availability of disposable local anesthetic infusion systems, and encouraging results from these early studies, has led to increasing popularity of these techniques for pain control in the post-discharge period. ${ }^{23}$ However, the clear benefits of these approaches for managing pain after orthopedic surgery must be balanced against the cost of the equipment and the resources need to safely manage these systems outside the hospital environment.

A critical assessment of the peer-reviewed literature regarding the optimal analgesic therapies for outpatient laparoscopic cholecystectomy by Bisgaard ${ }^{26}$ concluded that a "multimodal analgesic regimen consisting of a preoperative single dose of dexamethasone, incisional local anesthetics at the beginning and/or end of surgery) and continuous treatment with NSAIDs (or COX-2 inhibitors) during the first three to four days provided the best clinical outcome." These authors have further suggested that elimination of opioid-based analgesia would be highly desirable in the future. These important findings have been recently confirmed by White et al. ${ }^{14}$ In our prospective study involving the administration of celecoxib on the day of surgery and the subsequent three days after outpatient laparoscopic surgery as part of a multimodal analgesic regimen, we found that these patients not only experienced less pain and reduced need for opioid-containing oral analgesics, but more importantly, patients were able to resume normal activities of daily living one-to-two days earlier.

With the more widespread use of multimodal perioperative analgesic regimens involving both opioid and non-opioid analgesic therapies, physicians and nurses are becoming increasingly aware of the important role these techniques play in facilitating the recovery process and improving patient satisfaction. In a retrospective review, Pavlin et al. ${ }^{2}$ demonstrated the importance of controlling postoperative pain in order to facilitate recovery after ambulatory surgery. Moderate-to-severe pain prolonged the recovery room stay by $40-80$ min, and the adjunctive use of local anesthetics and NSAIDs decreased pain scores and facilitated an earlier discharge. However, until recently, there have been only a handful of well-controlled clinical studies documenting the beneficial effect of these multimodal therapeutic approaches with respect to important recovery variables [e.g., resumption of normal activities (e.g., dietary intake, bowel function), return to work]. ${ }^{12-15}$ Recently, Reuben et al. ${ }^{27,28}$ demonstrated the value of a multimodal analgesic regimen on both short and long-term outcomes after major outpatient orthopedic procedures. Although many factors, in addition to pain, must be carefully controlled in order to minimize postoperative morbidity and facilitate the recovery process after elective surgery (e.g., postoperative nausea and vomiting, hydration status), ${ }^{5}$ the adequacy of pain control remains a major concern for all patients undergoing elective surgical procedures, as well as the healthcare providers responsible for their care. ${ }^{7,16}$

In conclusion, the administration of 'preventative' multimodal analgesic techniques, during and after ambulatory surgery, can improve pain management by reducing pain scores, decreasing the need for opioid analgesics (and their uncomfortable side effects), as well as facilitating earlier mobilization and rehabilitation (i.e., resumption of normal activities), leading to greater patient satisfaction with the recovery process. Current evidence suggests that these improvements in patient outcome can best be achieved by using a combination of preventative analgesic techniques involving both central and peripheral-acting analgesic drugs, as well as novel approaches to administering these drug combinations in locations remote from the hospital.

\section{La prise en charge de la douleur après une chirurgie ambula- toire : où est-ce que ça coince ?}

Malgré les progrès récents apportés à notre connaissance des traitements analgésiques multimodaux pour 
la prise en charge de la douleur, ${ }^{1}$ nombreux sont les patients subissant des chirurgies ambulatoires qui ressentent encore des niveaux inacceptables de douleur après leurs opérations. ${ }^{2-4} \mathrm{La}$ douleur postopératoire est un symptôme complexe et multifactoriel qui nécessite une approche réfléchie utilisant plusieurs modalités de traitement avant d'obtenir des résultats optimaux quant au confort du patient et à un processus de rétablissement facilité. L'analgésie multimodale (ou « équilibrée ») constitue une approche de prévention de la douleur postopératoire qui se base sur l'administration d'une combinaison d'analgésiques opiacés et non opiacés, lesquels agissent sur différents sites des systèmes nerveux central et périphérique afin d'éliminer les effets secondaires liés à l'utilisation des opiacés. ${ }^{1,5}$ L'arsenal thérapeutique analgésique actuel pour la prise en charge de la douleur postopératoire ne cesse de croître. Cependant, il semble y avoir un décalage considérable entre la publication d'études portant sur l'analgésie dans la littérature révisée par les pairs qui suggèrent des approches pour améliorer la prise en charge de la douleur aiguë et l'application de ces concepts dans la pratique clinique. ${ }^{6}$

Une partie du problème est liée au fait qu'un nombre croissant d'opérations non urgentes et de plus en plus complexes sont réalisées dans un contexte ambulatoire (ou de séjour de courte durée) ; en effet, dans le cas de ces opérations, le recours à une analgésie contrôlée par le patient (ACP) avec des opiacés intraveineux ou aux techniques d'analgésie centrale neuraxiale (rachidienne et péridurale) n'est tout simplement pas pratique pour la prise en charge de la douleur aiguë. Cette population croissante de patients nécessite un régime analgésique périopératoire agressif qui procure un soulagement efficace de la douleur, a un minimum d'effets secondaires, est intrinsèquement sécuritaire et peut être géré par les patients et leur famille loin d'un centre hospitalier ou chirurgical.

Le soulagement de la douleur postopératoire est l'un des facteurs les plus importants quand vient le temps de déterminer si un patient peut recevoir son congé d'un centre chirurgical ; en outre, cela a également une influence prépondérante sur la capacité du patient à reprendre ses activités quotidiennes. ${ }^{3}$ L'analgésie périopératoire a traditionnellement été procurée à l'aide d'analgésiques opiacés (morphiniques) puissants. Toutefois, une trop grande dépendance envers la médication à base d'opiacés pour la prise en charge de la douleur aiguë est associée à plusieurs complications périopératoires [par ex., dépression respiratoire, somnolence et sédation, nausées et vomissements postopératoires (NVPO), prurit, rétention urinaire, occlusion intestinale, constipation], complications qui peuvent retarder le congé de l'hôpital et le retour aux activités quotidiennes. ${ }^{6}$

Les anesthésiologistes privilégient de plus en plus une combinaison d'agents analgésiques non opiacés comme traitement de 'première intention' pour prévenir la douleur dans la période postopératoire. Néanmoins, les analgésiques opiacés vont probablement rester l'option de traitement préférée pour les patients nécessitant un traitement analgésique «de secours » en période postopératoire et ce, jusqu'à ce que des analgésiques non opiacés plus puissants et à action plus rapide soient disponibles pour un usage clinique de routine.

En 2000, dans le but d'améliorer le contrôle de la douleur aiguë, l'organisme d'accréditation des hôpitaux américains JCAHO (Joint Commission on Accreditation of Healthcare Organizations) a introduit de nouvelles normes rendant obligatoire l'évaluation et le traitement de la douleur dans le cadre des soins de routine prodigués aux patients. Malheureusement, plusieurs institutions médicales ont mal interprété cette directive comme exigeant que le traitement de la douleur soit guidé par l'intensité de la douleur telle que rapportée par le patient sur une échelle numérique. ${ }^{7}$ À la suite de la mise en place d'un système numérique de gradation de la douleur dans leur salle de réveil, Frasco et coll. ${ }^{8}$ rapportent une augmentation considérable de l'utilisation d'analgésiques opiacés. Vila et coll..$^{9}$ ont rapporté que, en conséquence de la directive concernant la prise en charge de la douleur mise en place par le JCAHO, l'incidence d'effets secondaires liées à l'utilisation d'opiacés est passée de 11 à 25 par 100000 patients-jours d'hospitalisation dans leur centre médical. La plupart du temps, la survenue d'effets secondaires aux médicaments étaient précédés d'une réduction documentée du niveau de conscience du patient en raison d'une sédation provoquée par un opiacé. Dans le contexte ambulatoire, le premier facteur responsable des nausées et vomissements survenant après le congé hospitalier est l'administration orale d'analgésiques contenant des opiacés. ${ }^{10}$ Selon Raeder et coll., ${ }^{\text {l1 }}$ si l'on compare l'utilisation d'une thérapie orale combinée d'acétaminophène et de codéine à l'administration d'ibuprofêne, l'ibuprofêne est associée à moins d'effets secondaires gastro-intestinaux (par ex., NVPO, constipation) après une chirurgie ambulatoire.

Des études observationnelles ont confirmé qu'une douleur mal prise en charge est un facteur important qui contribue à retarder le congé de l'hôpital après une chirurgie ambulatoire. ${ }^{2,4}$ L'amélioration du contrôle de la douleur postopératoire permettra d'accélérer le retour à la une qualité de vie normale du patient 
ainsi que le retour à une fonctionnalité normale. ${ }^{5}$ Il a été suggéré que la clé de l'amélioration du processus de récupération après une chirurgie ambulatoire est l'adaptation des techniques analgésiques multimodales (ou «équilibrées») en tant qu'approches standard pour la prévention et le traitement de la douleur dans le contexte ambulatoire. ${ }^{1}$ Parmi les premières études à évaluer les approches permettant de faciliter le processus de récupération, plusieurs ont démontré que le recours à des techniques analgésiques multimodales pouvait améliorer une récupération précoce ainsi que d'autres pronostics importants au niveau clinique après une chirurgie ambulatoire. ${ }^{12,13}$ Ces avantages ont été confirmés dans des études plus récentes, ${ }^{14,15}$ et les techniques multimodales sont, à l'heure actuelle, la pratique recommandée dans les programmes de soins cliniques rapides. ${ }^{5}$ Il est clair que l'usage unique d'une modalité analgésique non opiacée [par ex., analgésiques locaux, anti-inflammatoires non stéroïdiens (AINS) et/ou acétaminophène] ne suffit pas à contrôler une douleur postopératoire modérée à sévère, et un usage trop important d'analgésiques opiacés génère trop d'effets secondaires indésirables. ${ }^{6,7}$

Les bienfaits à court et à long terme du recours à des régimes multimodaux d'analgésie pour réduire les effets secondaires associés à l'utilisation d'opiacés demeurent quelque peu controversés, parce la définition d'une "analgésie multimodale " n'est pas uniforme dans la littérature anesthésique et chirurgicale. ${ }^{1}$ Dans certains contextes, l'analgésie multimodale fait référence à l'administration systémique d'agents analgésiques présentant différents mécanismes d'action, alors que dans d'autres situations, le terme se rapporte à l'application concomitante d'une pharmacothérapie analgésique et d'une anesthésie régionale. Cependant, la lacune principale de plusieurs des études publiées réside dans le fait que les traitements analgésiques multimodaux ont été interrompus après le congé de l'hôpital. ${ }^{16}$ Par exemple, l'administration périopératoire d'inhibiteurs de la cyclo-oxygénase-2 (COX-2) dans le cadre d'un régime analgésique multimodal chez des patients externes subissant une cure d'hernie inguinale a procuré des bienfaits limités au-delà des premiers jours postopératoires. ${ }^{17}$ Mais si les inhibiteurs de la COX-2 sont administrés durant 3 à 5 jours après la chirurgie ambulatoire, ${ }^{14,15}$ les bienfaits concernant les devenirs de patients pertinents au niveau clinique s'étendaient bien au-delà de la simple (et prévisible) réduction de la douleur et du besoin de médicaments analgésiques à base d'opiacés.

Bien qu'il soit fort probable que les analgésiques opiacés continuent de jouer un rôle prépondérant dans le traitement aigu de la douleur modérée à sévère dans la période postopératoire immédiate, il est tout aussi probable qu'à l'avenir les analgésiques non opiacés prennent un rôle plus important en qualité d'analgésiques "de prévention ", étant donné que le nombre de chirurgies très peu invasives (dites 'par le trou de la serrure') ne cesse de croître. En plus des anesthésiques locaux et des AINS conventionnels, les inhibiteurs de la COX-2, l'acétaminophène, la kétamine, la dextrométhorphane, les agonistes alpha-2, la gabapentine, la prégabaline, les bêta-bloqueurs et les stéroïdes glucocorticoïdes peuvent tous procurer des effets bénéfiques lorsqu'ils sont administrés à une dose adéquate dans le cadre d'un régime analgésique multimodal dans un contexte périopératoire. ${ }^{1,6}$ Bien que les effets d'anesthésique local de la lidocaïne soient bien connus, les effets bénéfiques potentiels de la lidocaïne intraveineuse pour réduire la douleur postopératoire et faciliter le processus de récupération n'ont été que très récemment décrits dans la littérature anesthésique. ${ }^{18}$ On a également découvert que de nouvelles molécules tels que le capsicum produisaient des effets analgésiques prolongés en raison de leur capacité à modifier le stimulus nociceptif au niveau de la terminaison nerveuse périphérique. ${ }^{19}$

Il a été suggéré que les techniques analgésiques préventives procurent une meilleure analgésie en prévenant la mise en place d'une sensibilisation centrale. ${ }^{20}$ Malheureusement, il semble que cette approche n'offre pas plus d'avantages significatifs du point de vue clinique par rapport aux régimes multimodaux dits de prévention, qui comprennent une approche efficace de la prise en charge de la douleur amorcée au début de la période postopératoire et continuée au-delà du congé de l'hôpital. ${ }^{21}$ L'étude récente de Sun et coll. ${ }^{21}$ n'a pas réussi à démontrer un bienfait supplémentaire lorsqu'une dose préopératoire de célécoxib, inhibiteur de la COX-2, est administrée par rapport à une simple administration du médicament pour réaliser une analgésie préventive dans la période postopératoire. Le prolongement d'un régime thérapeutique analgésique multimodal dans la période postopératoire (et après le congé de l'hôpital) est clairement plus important pour l'amélioration de la prise en charge de la douleur qu'une dépendance exclusive envers les techniques analgésiques préventives.

Il est essentiel d'éduquer les patients et leurs familles (les personnes soignantes) au sujet de l'importance de continuer à prendre les médicaments analgésiques après le congé de l'hôpital et/ou du centre chirurgical de jour si nous voulons améliorer la qualité du contrôle de la douleur et faciliter la récupération future. L'attention récemment portée sur les effets secondaires associés aux opiacés en tant qu'entraves à 
un degré élevé de satisfaction du patient et à un congé donné rapidement après une chirurgie a également ajouté à l'intérêt du recours à des techniques anesthésiques locales (et régionales) pendant et après une chirurgie ambulatoire. ${ }^{6,7}$ Par exemple, les techniques d'anesthésie locale continues (par ex., blocs périneuraux, infiltrations au site d'incision) sont de plus en plus utilisées en raison de leur capacité à contrôler une douleur modérée à sévère après des interventions chirurgicales ambulatoires importantes. ${ }^{22-25}$ Des systèmes de perfusion jetables d'anesthésique local sont maintenant disponibles et les premières études ont révélé des résultats prometteurs. Ceci a mené à la popularité croissante de ces techniques pour le contrôle de la douleur après le congé de l'hôpital. ${ }^{23}$ Mais les avantages évidents de ces approches pour la prise en charge de la douleur après une chirurgie orthopédique mis en regard les coûts du matériel et les ressources nécessaires pour une prise en charge sécuritaire de ces systèmes à l'extérieur d'un contexte hospitalier.

Bisgaard $^{26}$ a mené une évaluation critique de la littérature révisée par des pairs concernant les thérapies analgésiques optimales pour la cholécystectomie par laparoscopie dans un contexte ambulatoire. Selon ses conclusions, " un régime analgésique multimodal consistant en une dose préopératoire unique de dexaméthasone, des anesthésiques locaux au site de l'incision au début et/ou à la fin de la chirurgie, ainsi qu'un traitement continu à base d'AINS (ou d'inhibiteurs de la $\mathrm{COX}-2$ ) pendant les premiers trois à quatre jours a procuré le meilleur résultat clinique. » En outre, les auteurs suggèrent que l'élimination de l'analgésie par des agents opiacés serait très désirable à l'avenir. Ces découvertes importantes ont été confirmées récemment par White et coll. ${ }^{14}$ Dans notre étude prospective impliquant l'administration de célécoxib le jour de la chirurgie et les trois jours suivants après une chirurgie ambulatoire par laparoscopie dans le cadre d'un régime analgésique multimodal, nous avons découvert que les patients ainsi traités non seulement ressentaient moins de douleur et avaient moins besoin d'analgésiques oraux contenant des opiacés, mais aussi et surtout, que les patients étaient capables de reprendre leurs activités quotidiennes un à deux jours plus tôt.

Le recours à des régimes analgésiques périopératoires multimodaux à base de thérapies analgésiques opiacées et non opiacées est de plus en plus courant; pour cette raison, les médecins et le personnel soignant prennent de plus en plus conscience du rôle prépondérant que ces techniques jouent pour faciliter le processus de récupération et pour améliorer la satisfaction du patient. Dans un compte-rendu rétrospectif, Pavlin et coll. ${ }^{2}$ ont démontré l'influence du contrôle de la douleur post- opératoire sur le temps et la qualité de la récupération après une chirurgie ambulatoire. Une douleur modérée à sévère prolongeait le séjour en salle de réveil de 40 à 80 minutes, et l'adjonction d'anesthésiques locaux et d'AINS diminuait les scores de douleur et favorisait le congé précoce du patient. Jusqu'à récemment, il n'y a toutefois eu qu'une poignée d'études cliniques bien contrôlées rendant compte de l'effet bénéfique de ces approches thérapeutiques multimodales sur les principales variables de récupération [par ex., reprise des activités quotidiennes normales (par ex., apport alimentaire, fonction intestinale), retour au travail]. ${ }^{12-15}$ Récemment, Reuben et coll. ${ }^{27,28}$ ont démontré les avantages d'un régime analgésique multimodal sur les devenirs à court et à long terme après des chirurgies orthopédiques importantes réalisées en ambulatoire. Bien que de nombreux facteurs doivent être contrôlés en plus de la douleur pour minimiser la morbidité postopératoire et faciliter le processus de récupération après une chirurgie non urgente (par ex., nausées et vomissements postopératoires, état d'hydratation), ${ }^{5}$ le soulagement de la douleur demeure une importante préoccupation de tous les patients subissant des chirurgies non urgentes, ainsi que des prestataires de soins de santé responsables de leur bien-être. ${ }^{7,16}$

En conclusion, l'administration de techniques analgésiques multimodales " préventives » pendant et après une chirurgie ambulatoire peut améliorer la prise en charge de la douleur en réduisant les scores de douleur, en diminuant le besoin en analgésiques opiacés (et leurs effets secondaires désagréables) et en facilitant une mobilisation et une réhabilitation plus précoce (c.à-d. une reprise des activités normales), ce qui a pour résultat une plus grande satisfaction du patient dans le processus de récupération. Les données actuelles suggèrent que le meilleur moyen de réaliser ces améliorations dans le devenir des patients consiste à utiliser une combinaison de techniques analgésiques préventives à base d'agents analgésiques agissant sur les systèmes nerveux central et périphérique ainsi que des approches innovantes dans l'administration de ces combinaisons de médicaments à l'extérieur de l'hôpital.

\section{References}

1 White PF. Multimodal pain management-the future is now! Curr Opin Investig Drugs 2007; 8: 517-8.

2 Pavlin DJ, Chen C, Penazola DA, Polissar NL, Buckley FP. Pain as a factor complicating recovery and discharge after ambulatory surgery. Anesth Analg 2002; 95: 627-34.

3 Chung F, Ritchie E, Su J. Postoperative pain in ambulatory surgery. Anesth Analg 1997; 85: 808-16.

4 Brennan F, Carr DB, Cousins M. Pain management: a 
fundamental human right. Anesth Analg 2007; 105: 205-21.

5 White PF, Keblet H, Neal JM, Schricker T, Carr DB, Carli F; Fast-Track Surgery Study Group. The role of the anesthesiologist in fast-track surgery: from multimodal analgesia to perioperative medical care. Anesth Analg 2007; 104: 1380-96.

6 White PF. The changing role of non-opioid analgesic techniques in the management of postoperative pain. Anesth Analg 2005; 101: S5-22.

7 White PF, Keblet H. Improving pain management: are we jumping from the frying pan into the fire? (Editorial). Anesth Analg 2007; 105: 10-2.

8 Frasco PE, Sprung J, Trentman TL. The impact of the joint commission for accreditation of healthcare organizations pain initiative on periooperative opiate consumption and recovery room length of stay. Anesth Analg 2005; 100: 162-8.

9 Vila H Jr, Smith RA, Augustyniak MJ, et al. The efficacy and safety of pain management before and after implementation of hospital-wide pain management standards: is patient safety compromised by treatment based solely on numerical pain ratings? Anesth Analg 2005; 101: 474-80.

10 White PF. Prevention of postoperative nausea and vomiting: A multimodal solution to a persistent problem. $\mathrm{N}$ Engl J Med 2004; 350: 2511-2.

11 Raeder JC, Steine S, Vatsgar TT. Oral ibuprofen versus paracetamol plus codeine for analgesia after ambulatory surgery. Anesth Analg 2001; 92: 1470-2.

12 Michaloliakou C, Chung F, Sharma S. Preoperative multimodal analgesia facilitates recovery after ambulatory laparoscopic cholecystectomy. Anesth Analg1996: 82: 44-51.

13 Eriksson H, Tenhunen A, Korttila K. Balanced analgesia improves recovery and outcome after outpatient tubal ligation. Acta Anaesthesiol Scand 1996; 40: 151-5.

14 White PF, Sacan O, Tufanogullari B, Eng $M$, Nuangchamnong N, Ogunnaike B. Effect of shortterm postoperative celecoxib administration on patient outcome after outpatient laparoscopic surgery. Can J Anesth 2007 54; 342-8.

15 Gan TJ, Joshi GP, Viscusi E, et al. Preoperative parenteral parecoxib and follow-up oral valdecoxib reduce length of stay and improve quality of patient recovery after laparoscopic cholecystectomy surgery. Anesth Analg 2004; 98: 1665-73.

16 White PF, Kehlet H. Postoperative pain management and patient outcome: time to return to work! (Editorial). Anesth Analg 2007; 104: 487-90.

17 Ma H, Tang J, White PF, et al. Perioperative rofecoxib improves early recovery after outpatient herniorrhaphy. Anesth Analg 2004; 98: 970-5.
18 Kaba A, Laurent SR, Detroz BJ, et al. Intravenous lidocaine infusion facilitates acute rehabilitation after laparoscopic colectomy. Anesthesiology 2007; 106: 11-8.

19 Kim KS, Nam YM. The analgesic effects of capsicum plaster at the Zusanli point after abdominal hysterectomy. Anesth Analg 2006; 103: 709-13.

20 Moiniche S, Keblet H, Dabl JB. A qualitative and quantitative systematic review of preemptive analgesia for postoperative pain relief: the role of analgesia. Anesthesiology 2002; 96: 725-41.

21 Sun T, Sacan O, White PF, Coleman J, Rohrich R, Kenkel JM. Perioperative vs postoperative celecoxib on patient outcome after major plastic surgery procedures. Anesth Analg 2008; 106: 950-8.

22 White PF, Issioni T, Skrivanek GD, Early JS, Wakefield. The use of a continuous popliteal sciatic nerve block after surgery involving foot and ankle: does it improve the quality of recovery? Anesth Analg 2003; 97: 13039.

23 Ilfeld BM, Enneking FK. Continuous peripheral nerve blocks at home: a review. Anesth Analg 2005; 100: 1822-33.

24 Capdevila X, Dadure C, Bringuier S, et al. Effect of patient-controlled perineural analgesia on rehabilitation and pain after ambulatory orthopaedic surgery: a multicenter randomized trial. Anesthesiology 2006; 105: 566-73.

25 Liu SS, Richman JM, Thirlby RC, Wu CL. Efficacy of continuous wound catheters delivering local anesthetic for postoperative analgesia: a quantitative and qualitative systematic review of randomized controlled trials. J Am Coll Surg 2006; 203: 914-32.

26 Bisgaard T. Analgesic treatment after laparoscopic cholecystectomy: a critical assessment of the evidence. Anesthesiology 2006; 104: 835-46.

27 Reuben SS, Ekman EF, Charron D. Evaluating the analgesic efficacy of administering celecoxib as a component of multimodal analgesia for outpatient anterior cruciate ligament reconstruction surgery. Anesth Analg 2007; 105: 222-7.

28 Reuben SS, Ekman EF. The effect of initiating a preventive multimodal analgesic regimen on long-term patient outcomes for outpatient anterior cruciate ligament reconstruction surgery. Anesth Analg 2007; 105: 228-32. 